\title{
Slice \& Dice: Identifying and Removing Bright Galactic Binaries from LISA Data
}

\author{
Louis J. Rubbo*, Neil J. Cornish ${ }^{\dagger}$ and Ronald W. Hellings ${ }^{\dagger}$ \\ ${ }^{*}$ Center for Gravitational Wave Physics, Pennsylvania State University, University Park, PA 16802 \\ ${ }^{\dagger}$ Department of Physics, Montana State University - Bozeman, Bozeman, MT 59717
}

\begin{abstract}
Here we describe a hierarchal and iterative data analysis algorithm used for searching, characterizing, and removing bright, monochromatic binaries from the Laser Interferometer Space Antenna (LISA) data streams. The algorithm uses the $\mathscr{F}$-statistic to provide an initial solution for individual bright sources, followed by an iterative least squares fitting for all the bright sources. Using the above algorithm, referred to as Slice \& Dice, we demonstrate the removal of multiple, correlated galactic binaries from simulated LISA data. Initial results indicate that Slice \& Dice may be a useful tool for analyzing the forthcoming LISA data.
\end{abstract}

\section{INTRODUCTION}

Inside the Milky Way galaxy there is a plethora of binaries whose emitted gravitational waves have a frequency inside the Laser Interferometer Space Antenna (LISA) band [1, $2,3,4]$. For the vast majority of these sources, the measured signals will be buried in the instrument noise. However, thousands of sources will have signals strong enough to be detected above the instrument noise at low frequencies $(0.1 \lesssim f \lesssim 3 \mathrm{mHz})$. The superposition of these sources will form a confusion limited background below which individual systems cannot be distinguished from the collective population. This confusion background will act as an additional noise component for the detector.

Lying above the confusion background will be a number of individual sources whose signals are stronger than the local rms value of the background [5]. Due to their relative brightness, they will be resolvable within the LISA data streams. Additionally, at higher frequencies, where the confusion background drops below the detector noise, individual sources will also be resolvable. Due to the large orbital periods and low chirp masses associated with galactic binaries, radiation reaction effects will not drive the binaries to coalescence during the mission lifetime, so their signals will be ever present in the detector output. It is these galactic binaries that are prime targets for data analysis techniques used for identification, characterization, and subtraction.

Even though these target signals are relatively bright, significant correlations may still exist between them. Consequently, sophisticated data analysis techniques capable of resolving the individual systems will be required. Here we present the Slice \& Dice algorithm which was developed to resolve the bright galactic binary signals that lie above the confusion limited background. Slice \& Dice uses the $\mathscr{F}$-statistic (a template based routine [6]) to identify and initially characterize the bright signals. The $\mathscr{F}$ statistic results are then used to initiate a least squares fitting procedure which refines 


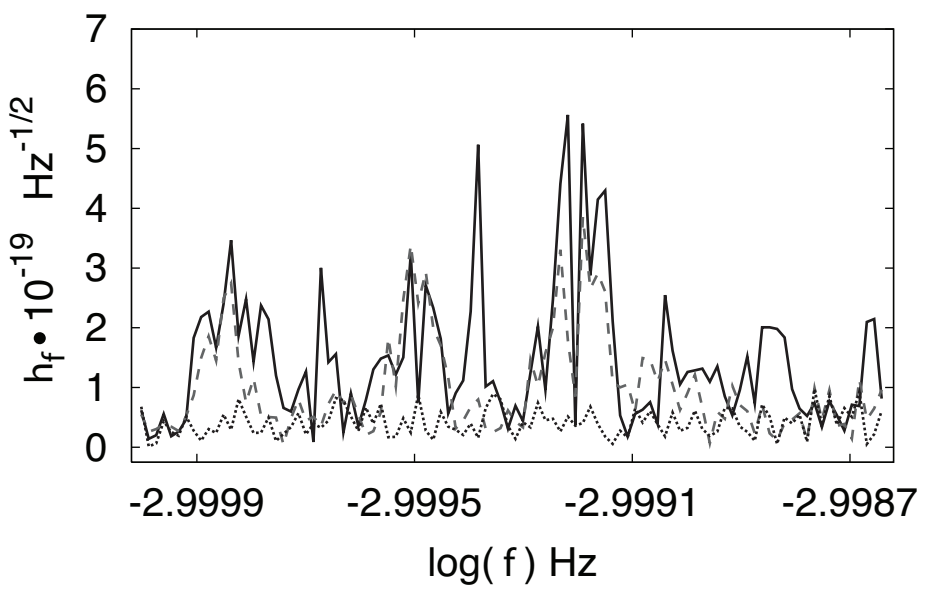

FIGURE 1. Results of a sequential identification, ordered by frequency, followed by a global removal scheme. The solid line is the original data, the dashed is the residue, and the dotted is the instrument noise.

the parameter estimates.

The rest of this paper proceeds as follows. In section 2 we discuss the motivation for Slice \& Dice by illustrating the importance of when signals are identified and subtracted from the data. In section 3 we describe the Slice \& Dice algorithm in detail and give a simple example of its use. Finally in section 4 we discuss future developments.

\section{IDENTIFICATION AND SUBTRACTION ORDER}

Template matching and least squares fitting provide a robust approach to identifying and subtracting individual signals from LISA's data [7]. However, when dealing with multiple signals simultaneously the order of identification and removal is crucial.

Figure 1 shows the spectral amplitudes for a sequential identification, ordered by frequency, followed by a global removal using a template matching scheme on twenty signals within one hundred frequency bins ${ }^{1}$ added to Gaussian noise. The solid line is the original data, the dotted is the instrumental noise, and the dashed is the residue after subtraction. The large residue indicates that this approached failed to accurately identify the injected signals. The failure arose because the algorithm treats the other comparable, bright signals as noise while attempting to fit for one signal.

Figure 2 demonstrates the result of a sequential fit and subtraction procedure where the order of fitting and removal is based on the signal-to-noise ratios (SNRs). Although the residue looks reasonable, a correlation comparison of the fitted signals to the original injections indicates that the weaker signals were not properly identified. As before, the interference from the other bright signals as the algorithm attempts to fit for one signal

${ }^{1}$ A frequency bin $\Delta f$ is equal to one on the observation time, $\Delta f=T^{-1}$. For a one year observation, which is used here, $\Delta f=3.2 \times 10^{-8} \mathrm{~Hz}$. 


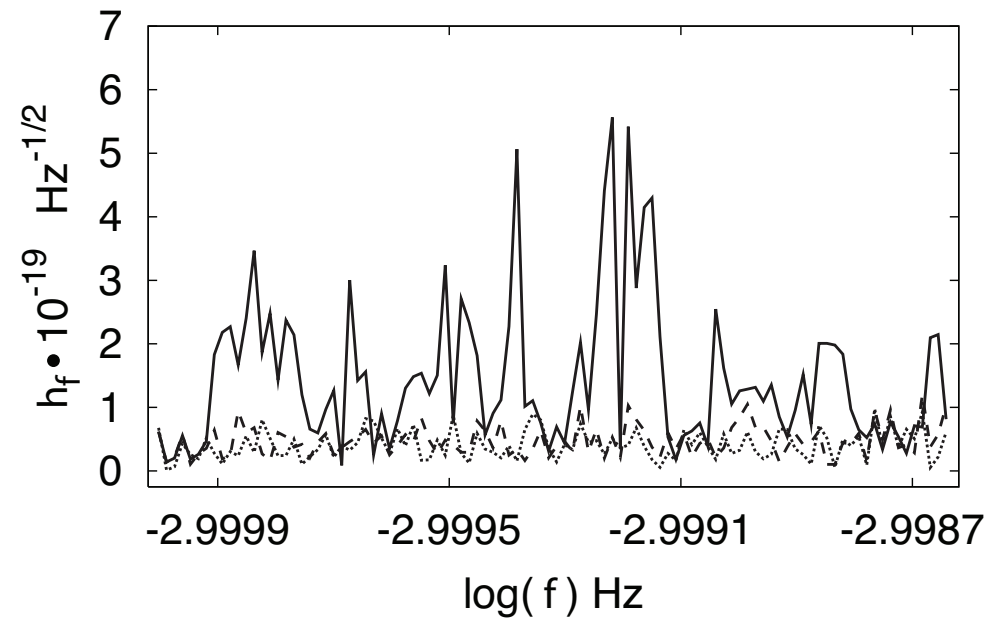

FIGURE 2. Results of a sequential identification and removal algorithm with the order is from highest SNR to the lowest. The line identifications are the same as Figure 1.

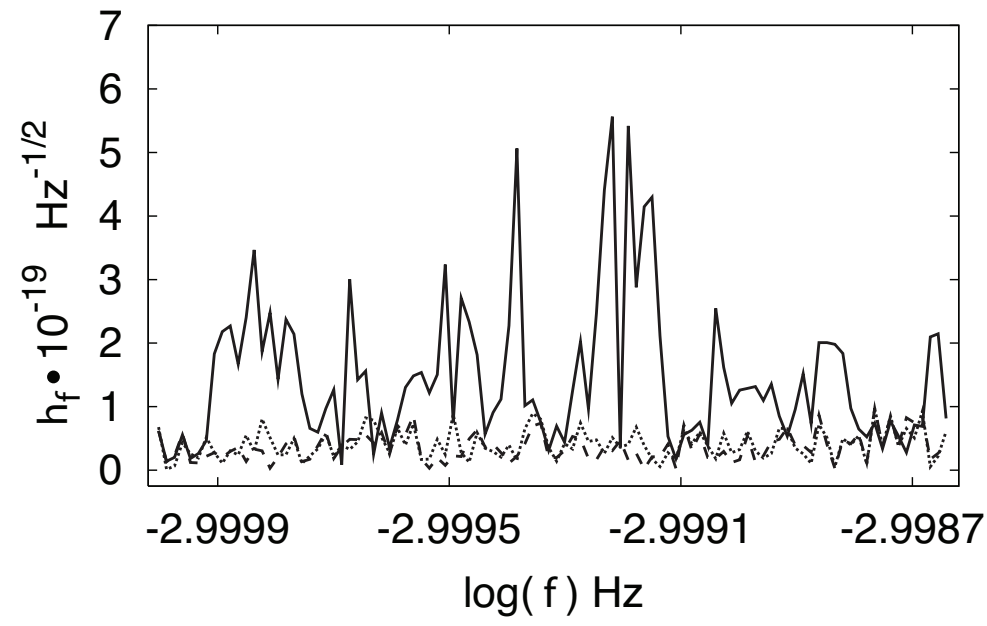

FIGURE 3. Results of a simultaneous identification procedure. The line identifications are the same as Figure 1.

causes errors, which ultimately propagate through to the fits for the weaker sources due to the intermediate subtractions.

Figure 3 demonstrates the result from performing a simultaneous fitting and removal scheme using a least squares fitting routine initiated at the true parameter values. In this case there are acceptable fits for each signal. However, for this approach to work we had to assume the initial values for the least squares procedure. Normally these values would be obtained from a search algorithm, such as a template matching routine. Unfortunately, when the number of bright systems increases, the resulting number of templates required to sufficiently cover the parameter space make a template approach impractical.

Slice \& Dice was developed to incorporate the robust attributes of template matching and least squares fitting while circumventing the problems of a large template bank and 
providing initial guesses for the least squares routine.

\section{SLICE \& DICE ALGORITHM}

The Slice \& Dice algorithm is an iterative routine that incorporates multiple LISA data streams. Each iteration involves the following steps,

1. An $\mathscr{F}$-statistic (i.e. a template based) search is used to find the brightest signal and return an initial estimate for its parameter values.

2. The $\mathscr{F}$-statistic parameter estimates, along with previous iteration estimates, are used to initiate a least squares fitting routine on the original data. The least squares routine simultaneously solves for $i$ signals, where $i$ is the number of sources found so far.

3. The least squares fitting routine is repeated until the change in the $\chi^{2}$ is insignificant $(<0.01)$. At each least squares cycle the initial parameter value guesses are supplied from the previous least squares iteration.

4. The estimated signals are subtracted from the original data streams and this partially cleaned data is used to initiate the next Slice \& Dice iteration.

These steps are repeated until each bright source $(\mathrm{SNR}>5)$ are identified and removed.

As an example of Slice \& Dice results, consider two systems with SNRs of 17 and 12, separated in frequency by $0.07 \Delta f$, in sky location by $90^{\circ}$, and with random orientations. The initial correlation matrix between the injected signals is

$$
r_{1 y r}=\left(\begin{array}{cc}
1 & 0.090 \\
0.090 & 1
\end{array}\right)
$$

While their frequencies are nearly identical, these sources are largely separated on the sky and therefore have small cross-correlations.

Figure 4 shows the spectral amplitudes before and after Slice \& Dice has been applied to the data, along with the instrument noise. The final comparison correlation matrix between the original and the estimated signals is

$$
r_{1 y r}=\left(\begin{array}{cc}
0.94 & 0.11 \\
0.06 & 0.99
\end{array}\right)
$$

Slice \& Dice was able to accurately identify the two signals.

\section{FUTURE DEVELOPMENTS}

At this time we are testing Slice \& Dice in order to understand its strengths and limitations. We have successfully applied the algorithm on bright source densities comparable to those shown in Figures 1 through 3 (i.e. one bright source every five frequency bins). However, possible parameter degeneracies and how their effects propagate through the 


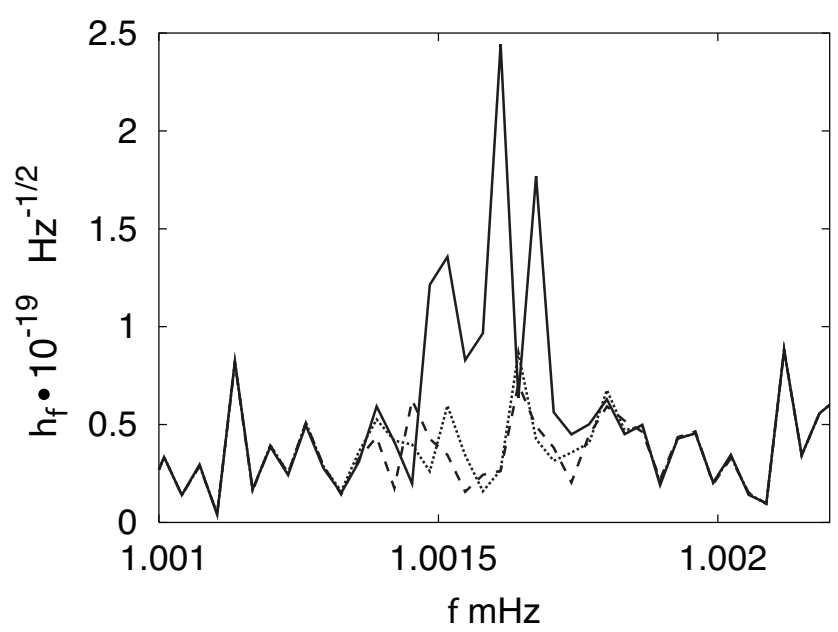

FIGURE 4. Results of Slice \& Dice on two signals with nearly identical frequencies but largely separated on the sky. The line identifications are the same as Figure 1.

iterative Slice \& Dice routine are not fully understood. We are currently testing how the algorithm responds to such degeneracies, and how they may limit its capabilities.

In addition to the above mentioned tests, we are also expanding the scope of applicability. Slice \& Dice is currently only applicable on a small segment of the LISA band. In the future we will expand and automate the algorithm so that it can take the full bandwidth of data. Additionally, we would like to compare and contrast Slice \& Dice's performance to other galactic binary search procedures such as gCLEAN [8], Markov Chain Monte Carlo methods [9, 10], genetic algorithms [11], and maximum entropy.

\section{ACKNOWLEDGMENTS}

The work by LJR was supported by the Center for Gravitational Wave Physics. The Center for Gravitational Wave Physics is supported by the NSF under Cooperative Agreement No. PHY 01-14375. NJC was supported by NASA grant NNG05GI69G.

\section{REFERENCES}

1. C. R. Evans, I. Iben Jr., and L. Smarr, ApJ 323, 129-139 (1987).

2. D. Hils, P. L. Bender, and R. F. Webbink, ApJ 360, 75-94 (1990).

3. G. Nelemans, L. R. Yungelson, and S. F. Portegies Zwart, A\&A 375, 890-898 (2001).

4. M. J. Benacquista, J. DeGoes, and D. Lunder, Class. Quantum Grav. 21, S509-S514 (2004).

5. S. E. Timpano, L. J. Rubbo, and N. J. Cornish, Phys. Rev. D 73, 122001 (2006).

6. P. Jaranowski, A. Królak, and B. F. Schutz, Phys. Rev. D 58, 063001 (1998).

7. R. W. Hellings, Class. Quantum Grav. 20, 1019-1029 (2003).

8. N. J. Cornish, and S. L. Larson, Phys. Rev. D 67, 103001 (2003).

9. N. J. Cornish, and J. Crowder, Phys. Rev. D 72, 043005 (2005).

10. R. Umstätter, et al., Phys. Rev. D 72, 022001 (2005).

11. J. Crowder, N. J. Cornish, and J. L. Reddinger, Phys. Rev. D 73, 063011 (2006). 